\title{
En torno a la unidad del tratado hopocrático Denatura hominis a la luz de la estadística lingüística ${ }^{1}$
}

\author{
Jesús Ángel y Espinós \\ Universidad Complutense de Madrid \\ espinos@filol.ucm.es
}

Recibido: 5 de abril de 2010

Aceptado: 30 de noviembre de 2010

\section{RESUMEN}

En este artículo se intenta aportar nuevos datos sobre la polémica en torno a la unidad del tratado hipocrático De natura hominis. Tras la erudita edición del texto griego a cargo de Jouanna (1975), la comunidad científica está de acuerdo en considerar que el tratado De natura hominis constituye una unidad que incluye los capítulos 16-22, también conocidos como De diaeta salubri. Sin embargo, en el presente trabajo se ha emprendido la tarea de reexaminar los diferentes aspectos de la controversia desde un nuevo punto de vista. Por lo tanto, se ha adoptado como punto de partida metodológico la aplicación de métodos estadísticos, tales como la prueba «t de Student-Fisher» o el análisis de correlación de rangos de Spearman. De acuerdo con los datos obtenidos en la investigación, basados en el estudio estadístico del asíndeton, de las conjunciones, de la parataxis y de la hipotaxis, los capítulos de la parte denominada De diaeta salubri deberían ser excluidos del tratado De natura hominis y considerados como una obra independiente.

Palabras clave: estadística lingüística, estudios sobre estructura y composición, Corpus Hippocraticum, De natura hominis

\begin{abstract}
In the present article the author would like to shed new light on the controversy about the unity of the Hippocratic treatise De natura hominis. After the erudite edition of the Greek text by Jouanna (1975), scholars generally agree that the treatise De natura hominis constitutes a compositional unity which includes the chapters 16-22, also known as De diaeta salubri. Nevertheless, the author of this paper has undertaken the task of re-examining the pros and cons of the debate from a new point of view. Consequently, he has taken as a methodological starting-point the application of Statistical Linguistics through the implementation of the Student-Fisher $t$ test and the Spearman's rank correlation coefficient. According to his research, based on the statistical study of asyndeton, conjunctions, parataxis and hypotaxis, the chapters of the so-called De diaeta salubri should be detached from the treatise De natura hominis and considered as an independent work.
\end{abstract}

Key words: Statistics in Language Studies, Studies on Structure and Composition, Corpus Hippocraticum, De natura hominis

${ }^{1}$ Este trabajo se inscribe en el proyecto FFI-2009-10118 (subprograma FILO) «Estudios sobre el Corpus Hippocraticum y su influencia», dirigido por el Dr. Ignacio Rodríguez Alfageme. 
El empleo de métodos estadísticos ha resultado especialmente productivo en el estudio del Corpus Hippocraticum, colección de textos que comprende tratados que abarcan un amplio espacio de tiempo y que defienden teorías diversas, si bien todos ellos se pueden enmarcar bajo la denominación, un tanto laxa, de medicina hipocrática. Así pues, tomando como punto de partida metodológico el empleo de recursos estadísticos hemos afrontado el estudio del tratado De natura hominis en un intento de aproximación a los textos de la manera más aséptica posible, intentando evitar en todo momento cualquier tipo de prejuicios o consideraciones previas que puedan mediatizar los resultados finales. ${ }^{2}$

\section{ESTRUCTURA Y UNIDAD DEL TRATADO DE NATURA HOMINIS}

A raíz de la edición con traducción y comentario del tratado De natura hominis por parte de Jouanna (1975) parece haberse llegado al consenso indiscutible de que este tratado consta no solamente de los quince capítulos editados por Littré (VI, pp. 32-69) bajo este título, sino también de nueve capítulos más que Littré publicó con el nombre de Du régime salutaire (VI, pp. 72-87) como pertenecientes a otra obra independiente. No obstante, los dos últimos capítulos se han de atetizar pues el penúltimo corresponde a De morbis II, 2 y el último al primer capítulo del tratado De affectionibus.

Littré fue el primer editor moderno que rompió la unidad del opúsculo y a partir de él hasta la edición de Jouanna (1975) se había respetado esta división, tal y como se aprecia en la edición de Jones (1931), que sigue la distribución así como la numeración de los capítulos de Littré, quien decidió numerar aparte el texto del Du régime salutaire.

Este texto, si se admite su unidad postulada por Jouanna aunque rechazada sin embargo por algunos estudiosos, ${ }^{3}$ constituiría el único ejemplo del Corpus Hippocraticum al que se le podría adscribir el nombre de un autor: Pólibo, discípulo $\mathrm{y}$, según la tradición, yerno de Hipócrates. ${ }^{4}$ No obstante, Galeno en su comentario al

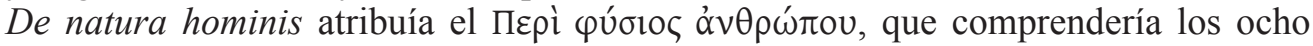
primeros capítulos en los que se expone la teoría de los cuatro humores, ${ }^{5}$ a Hipócrates,

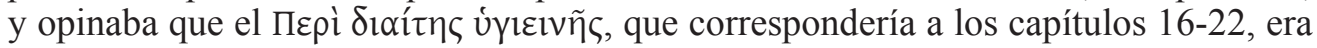
obra de Pólibo, mientras que el resto (capítulos 9-15) eran producto de la labor de un compilador helenístico, que habría reunido las dos obras en una para su propio provecho con el fin de venderlas a los Atálidas o a los Ptolomeos. ${ }^{6}$ Por otra parte,

\footnotetext{
${ }^{2}$ Entre los estudios que han tratado el Corpus Hippocraticum a partir de presupuestos similares hemos de citar los de Rodríguez Alfageme (1992), (1993), Ángel y Espinós (2003), Sierra de Grado (2003) y Labiano Ilundáin (2004).

${ }^{3}$ Sintomático de lo impreciso de la cuestión es la postura de Alsina i Clota quien pasó de una concepción claramente analítica (1974) a otra unitaria (1983).

${ }^{4}$ Sobre el parentesco entre Hipócrates y Pólibo, Jouanna (1969: 553) expresa serias dudas pues Galeno normalmente se refiere a Pólibo simplemente como un alumno de Hipócrates; por su parte, Grensemann (1974: col. 429) no está de acuerdo con Jouanna pues lo que prima en los escritos de Galeno es la relación maestro-alumno entre Hipócrates y Pólibo, no su parentesco familiar suegro-yerno.

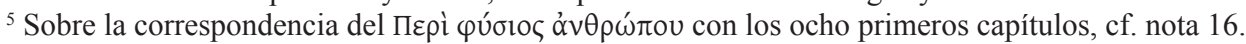

${ }^{6}$ Hippocratis De natura hominis liber et Galeni in eum commentarius II, ed. Kühn, vol. 15. 108,8 109,11 = Mewaldt 57, 6-16.
} 
la descripción de los vasos sanguíneos que aparece citada por Aristóteles, Historia animalium III, 3 (512b,12-513a,7), bajo el nombre de Pólibo es similar a la que se encuentra en De natura hominis, 11. Este testimonio, aun en contra de la adscripción de las distintas partes según Galeno, abogaría también por la autoría de Pólibo.

Poseemos igualmente otro testimonio de gran valor, si bien más reciente que el de Aristóteles pero que sin duda bebe de fuentes antiguas. Se trata del papiro 137, conservado en el British Museum y conocido como Anonymus Londinensis, cuya datación se sitúa en torno a los siglos I y II de nuestra Era. Los restos de las 39 columnas, llegadas hasta nosotros en estado fragmentario, contienen un resumen de teorías de diferentes médicos. Gracias a Galeno sabemos que las doctrinas médicas de la Antigüedad habían sido compiladas por Menón, discípulo de Aristóteles; así pues existe una cierta probabilidad de que el Anonymus Londinensis remonte en última instancia a la obra de Menón. Desafortunadamente, los datos del Anonymus Londinensis son contradictorios en relación con el juicio de Galeno pues cuando resume las palabras del capítulo 9 (Anon. Lond., col. VII 15, ed. Jones) del De natura hominis afirma que pertenecen a Hipócrates, pero sin embargo atribuye a Pólibo (Anon. Lond., col. XIX 1, ed. Jones) doctrinas que se relacionan con las que se encuentran en los capítulos 3 y 4 de dicho tratado. En suma, los testimonios de Aristóteles y del Anonymus Londinensis parecen atribuir a Pólibo los capítulos 3, 4 y 11, por lo que si el tratado constituyese una unidad, la autoría habría de adscribirse a este médico. ${ }^{7}$

Así pues, dejando al margen la posible autoría del tratado, la polémica en torno a la unidad del De natura hominis y De diaeta salubri merece ser tratada con suma atención, como paso preliminar para la investigación.

El opúsculo De diaeta salubri aparece en la tradición manuscrita como un apéndice del De natura hominis; de hecho, la suscripción que indica el final de la obra se sitúa tras los capítulos que Littré agrupa bajo el título de Du régime salutaire y no hay ninguna separación expresa entre ambos tratados. No obstante, los manuscritos ofrecen al inicio del capítulo 16 -que constituiría por esta causa el c. 1 del Du régime

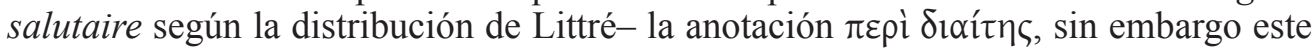
epígrafe no reviste mayor importancia pues es similar a otros que anuncian a veces

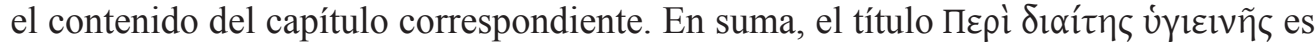
ajeno a la tradición manuscrita y remonta en última instancia a un pasaje de Galeno, ${ }^{8}$

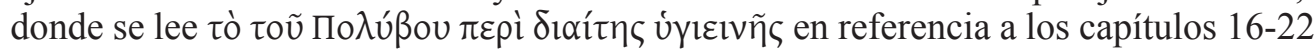
del tratado. ${ }^{9}$ Además, este texto demuestra que Galeno, a pesar de sus convicciones

\footnotetext{
${ }^{7}$ Como ya hemos señalado y más adelante se expondrá con más detalle, el De natura hominis se ha dividido en tres secciones ya desde Galeno; el problema reside en que los distintos autores no se han puesto de acuerdo en los límites de cada sección. Este desacuerdo ha surgido sobre todo a la hora de establecer las dos posibles secciones en que se pueden dividir los primeros quince capítulos; es decir, la parte del tratado que según algunas voces conformaría el De natura hominis. Respecto a los capítulos finales dedicados a la dieta (cc. 16-22), conocidos como De diaeta salubri, parece haber unanimidad en su extensión y en la necesidad de expurgar los cc. 23-24 por pertenecer a otros tratados.

${ }^{8}$ Hippocratis De natura hominis liber et Galeni in eum commentarius II, ed. Kühn, vol. 15. 109,5 = Mewaldt 57, $11 \mathrm{~s}$.

${ }^{9}$ El único testimonio en el que aparece este título se halla en el recentior Par. gr. $2142(\mathrm{H})$ del siglo

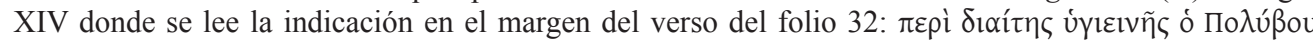


analíticas respecto al tratado, leía dentro del conjunto del De natura hominis la parte final consagrada al régimen.

Probablemente a lo largo de la Antigüedad ambas partes se leyeron juntas y no sería hasta el Renacimiento cuando, bajo el predicamento de Galeno, surgieron posturas analíticas, ${ }^{10}$ que quedarían refrendadas en época moderna por Littré y por Jones (1931), aunque no sin la oposición de Villaret (1911), Deichgräber (1933) ${ }^{11}$ y por supuesto Jouanna (1975).

Sin lugar a dudas, hasta la aparición de la edición del De natura hominis con comentario y traducción a cargo de Jouanna (Paris 1975), la obra más ambiciosa a propósito de este tratado la constituyen las Hippokratische Untersuchungen de Fredrich (Berlin 1899), quien propugna posturas analíticas bastante radicales en lo que se refiere a la estructura y unidad de la obra, así como a la autoría.

Fredrich está de acuerdo parcialmente con Galeno en que se trata de una obra en la que se han recogido varios textos, aunque disiente en la idea de un falsificador que habría reunido en beneficio propio dos tratados independientes pues de haber sido así, éste habría puesto más celo en su labor y en la calidad final. Asimismo, sostiene la tesis de que fue un médico quien quiso conservar en un único tratado una serie de notas preparadas para una lección o para uso personal, tomadas algunas de sus lecturas y otras de su propia redacción. En este úró $\mu v \eta \mu \alpha$ se habrían recogido disputas sobre el origen y la curación de las enfermedades en general (los ocho primeros capítulos que

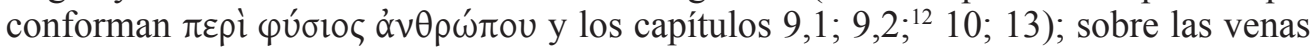
(c. 11); el pus (c. 12); la orina (c. 14); la fiebre (c. 15) y, finalmente, la dieta a excepción de los dos capítulos finales que son un añadido.

Por otra parte Fredrich divide el texto siguiendo pautas relacionadas con el contenido y la doctrina que se defiende en cada una de las secciones que presupone como obra de

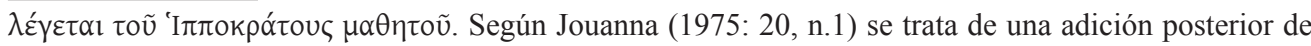
clara raigambre galénica por lo que carece de interés alguno.

${ }^{10}$ Sabemos que Mercurial en su edición griega con traducción latina del Corpus Hippocraticum (Venezia 1588) optó por separar ambas obras. La princeps aldina de Asulano (Venezia 1526) publicó ambos opúsculos como si de una sola obra se tratase, pero sin embargo en el índice se citaban los dos tratados por separado: el

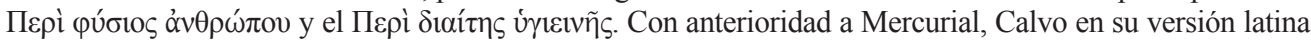
que precede en un año a la aldina (Roma 1525), añadió siguiendo una extraña disposición la parte consagrada al régimen al final del libro primero del tratado De victu. Igualmente, en la edición latina de Valgrisio (Venezia 1575), que corrió a cargo de varios eruditos, se publicaron por separado tras el De natura hominis los capítulos dedicados al régimen bajo el título de De diaeta salubri, siguiendo de nuevo la tradición galénica.

${ }^{11}$ En su libro dedicado a Epidemias, Deichgräber (1933: 111) sostiene la tesis de que los capítulos finales que versan sobre la dieta pertenecen al De natura hominis: «[...] zuletzt eine Kapitelreihe (sc. De diaeta salubri), in der man schon immer eine ursprünglich selbständige Schrift gesehen hat, eine Diätetik für Laien, die in Inhalt und Aufbau, wie man ebenfalls längst erkannt hat, zu den Eigentümlichkeiten von Kap. 1-9 restlos paßt.»

${ }^{12}$ Fredrich (1899: 16-21), en su afán analítico, divide por motivos temáticos el c. 9 en dos partes, que según él pertenecen al mismo redactor. En la segunda parte, que empieza a partir de $\alpha \dot{\imath} \delta \dot{\varepsilon}$ voṽ

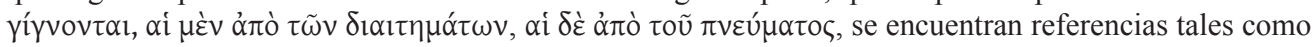

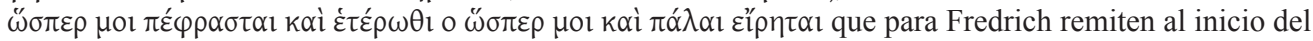
mismo capítulo razón por la que piensa que entre las dos partes del c. 9 quizás hubiera un texto perdido, pues de lo contrario el adverbio đó́ $\alpha$ estaría un tanto fuera de lugar. 
un mismo autor. De acuerdo con esta teoría considera que nos encontramos ante la labor

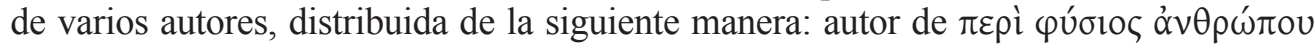
(cc. 1-8); autor de los capítulos 11, 12 y 14; autor de los capítulos 9,1, 9,2, 10, 13 y

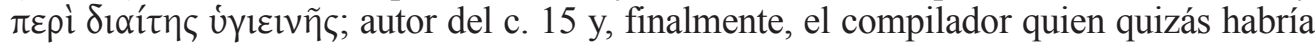
redactado el c. 15 y podría ser el autor de las interpolaciones en el texto, especialmente visibles por ejemplo en el c. 8, donde Fredrich propone atetizar algunos pasajes.

La crítica más mordaz a las tesis de Fredrich proviene de Jouanna (1975) quien en su edición con comentario y traducción del De natura hominis intenta desbaratar los puntos principales de Fredrich, así como de toda la escuela analítica en la que se incluyen Littré y Jones.

Jouanna (1975: 22-38) propone desde su punto de vista unitarista una división tripartita por motivos de contenido. Al igual que Jouanna ya Galeno había propugnado una división en tres partes, si bien para éste último la subdivisión conllevaba la existencia de tres autores distintos. Así pues Jouanna subdivide la obra en tres secciones formadas respectivamente por una parte teórica (cc. 1-7), una segunda consagrada a la patología y a la terapéutica (cc. 8-15) y finalmente una última dedicada al régimen (cc. 16-22, excluyéndose los cc. 23-24, que pertenecen a otros tratados del Corpus Hippocraticum). Esta división del texto se diferencia de la de Galeno en el hecho de que para el médico de Pérgamo la primera parte comprendería los primeros ocho capítulos, que

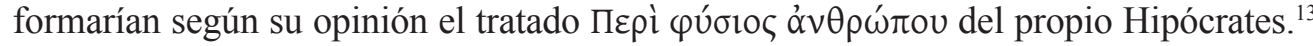
En su momento Fredrich (1899: 15 s.) sostuvo que los primeros ocho capítulos constituían una unidad temática y de autor pues consideraba el octavo capítulo como el final del desarrollo expositivo sobre la naturaleza del hombre, si bien para que su propuesta fuese perfectamente acorde con el texto transmitido era preciso eliminar dos pasajes que contradecían su opinión. A diferencia de Fredrich, Jouanna postula que el capítulo octavo no es una conclusión sino una transición que sirve para iniciar la nueva sección dedicada a cuestiones terapéuticas y patológicas tras haber concluido en el capítulo anterior la exposición general sobre la naturaleza humana. Al optar por esta tesis Jouanna también entra en disputa con la postura que en su momento adoptó Schoene (1900: 657) quien veía en el capítulo noveno - donde se expone una nueva etiología de la enfermedad, causada por elementos exteriores-, una continuación de la doctrina basada en la preponderancia de un humor sobre los otros que aparecía en los primeros ocho capítulos, los cuales a su parecer constituían una unidad. Por lo tanto, según Schoene los capítulos se agruparían de la siguiente manera: cc. 1-9, cc. 10-15 y cc. 16-22. ${ }^{14}$

\section{BASES TEÓRICAS DE LA PRESENTE INVESTIGACIÓN}

Como ya se ha señalado, el núcleo fundamental del presente trabajo se ha basado

${ }^{13}$ Fredrich (1899: 14) señala que Galeno consideraba que el tratado De natura hominis ocupaba las primeras 240 líneas del texto, lo que equivale a los ocho primeros capítulos según se puede comprobar

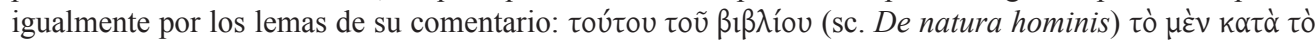

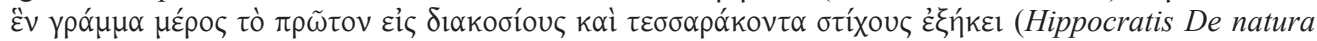
hominis liber primus et Galeni in eum commentarius, ed. Kühn, vol. 15. 9,17 s. = Mewaldt 7, 21 s.)

${ }^{14}$ Deichgräber (1933: 112), siguiendo a Schoene, acepta la misma disposición del texto. 
en el empleo de métodos estadísticos encaminados a discernir las posibles interrelaciones entre las diferentes secciones que parecen componer el texto. ${ }^{15}$

Dado que la estadística trabaja con datos numéricos hemos procedido a aislar algunos caracteres cuantificables para someter el texto a diferentes pruebas; en el presente estudio, los caracteres cuantificables que se han elegido han sido los nexos oracionales o, en su defecto, la ausencia de éstos, como es el caso del asíndeton.

De esta manera, se ha procedido a dividir el texto de las tres secciones que tradicionalmente conforman De natura hominis en grupos de 25 oraciones, computando las diferentes partículas de coordinación y las conjunciones subordinantes empleadas, al igual que las veces que la unión entre oraciones se realiza a través del asíndeton. El supuesto teórico del que parte esta cuantificación radica en que estos nexos oracionales son una característica constante en la lengua de un mismo autor y varían según los diversos autores y las épocas; por lo tanto, la comparación de los resultados de las diferentes partes permite conocer la distribución que se hace de los nexos oracionales (presentes o ausentes) en cada sección, lo cual posibilita la descripción del estilo así como la intención del autor y su predisposición e interés por cuestiones estilísticas.

Esta labor aparentemente sencilla presenta no pocas dificultades en su aplicación práctica y suscita problemas de difícil resolución en casos concretos. Así pues, es totalmente imprescindible la formulación de unos criterios a seguir en caso de duda respecto a cuándo un nexo (o su ausencia) introduce una oración o no. ${ }^{16}$

Así pues se ha considerado que la partícula $\mu \varepsilon ́ v$ introduce oraciones coordinadas; kaí sólo se ha visto reflejado en los cómputos finales cuando se comporta como conjunción propiamente dicha, no cuando presenta un valor adverbial o amplía un término de la misma oración. No obstante, koú se ha incluido en el recuento cuando coordina dos oraciones nominales puras.

Hay ciertas combinaciones que exigen un tratamiento especial: así en el caso de la

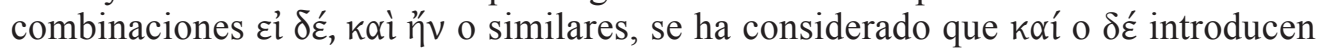
la oración principal mientras que $\eta \tilde{v}$, $\varepsilon i ̉$ o la conjunción correspondiente introducen la oración subordinada.

Por otra parte, el objetivo de este trabajo ha consistido en comparar las medias y la dispersión de los datos que ofrecen las distintas secciones del De natura hominis con el fin de relacionarlas o diferenciarlas entre sí, según la distribución obtenida mediante la

${ }^{15}$ Entre los programas que hoy en día se utilizan con mayor frecuencia en el campo de la estadística hemos de citar los siguientes: SAS (Statistical Analysis System), MINITAB y SPSS (Statistical Package for Social Sciences). Dentro de estos programas, el paquete informático SPSS es el más extendido en el ámbito académico por su facilidad de uso por lo que ha sido el sistema del que nos hemos servido en nuestro trabajo. No obstante, la estadística lingüística se ha venido empleando con fiabilidad antes de la era computacional. El ejemplo clásico de esta labor lo encontramos en la obra de Müller, Estadística lingüística (1973), que constituye una obra pionera pues marcó la pauta a seguir respecto a las pruebas más recurrentes que luego se impondrían en los estudios lingüísticos, como por ejemplo el test $\chi^{2}$ de Pearson, el test de Student-Fisher o los tests de rangos como el de Spearman o el de Bravais-Pearson. A propósito del SPSS, que ya ha alcanzado su versión 15.0 y utiliza como soporte el sistema operativo Windows, se puede consultar el libro de Rietveld y van Hout (2005).

${ }^{16}$ Sobre las dificultades que pueden surgir en la segmentación del texto, el trabajo de Sierra de Grado (2003: 405-407) ofrece un planteamiento muy detallado de los criterios a aplicar, criterios que en líneas generales coinciden con los nuestros. 
prueba estadística «t de Student-Fisher». Asimismo, se ha aplicado a los datos el cálculo del coeficiente de correlación de Spearman.

Para el análisis estadístico partimos de la base de que cada sección ha de ser interpretada como una muestra de la creación de un posible autor, entendiendo por muestra el grupo de sujetos en el que se recogen los datos y se realizan las observaciones, siendo realmente un subgrupo de la población accesible, población que se ha de interpretar como la obra de un mismo autor.

A su vez, la muestra se ha segmentado en grupos de 25 oraciones, que se han ido detallando según el orden de aparición de los distintos nexos oracionales registrados:

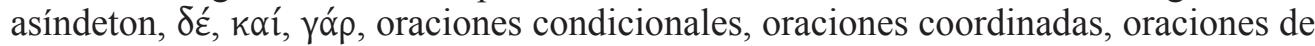
relativo y oraciones subordinadas.

Una vez reducidos los textos a datos cuantificables, se ha procedido a aplicar en primer lugar la prueba «t de Student-Fisher» con la ayuda del paquete estadístico SPSS. Esta prueba se aplica cuando las unidades que componen ambas muestras o al menos una de ellas $(n)$ es pequeño. En la práctica, se admite que un número de observaciones es «grande» a partir de $n \geq 30$, número que no se supera en ningún caso en la presente investigación.

En cada sección se han cuantificado unas determinadas medidas, que luego serán comparadas entre sí:

Media: Suma de todos los valores de cada grupo de 25 oraciones dividida entre el número total de unidades que componen la muestra $(\overline{\mathrm{x}})$.

Varianza de la muestra: Media de los cuadrados de las desviaciones por relación a la media $\left(\sigma^{2}\right)$.

Desviación típica: Raíz cuadrada de la varianza $(\sigma)$. Representa la amplitud de la dispersión de una distribución en torno a la clase central.

Error típico de la media: Medida de la dispersión que presentan las medias en la distribución muestral de dichas medias: $\sigma / \sqrt{ } n$

Posteriormente, para realizar la comparación entre dos secciones se han de utilizar las siguientes constantes y medidas de dispersión:

$N$ : Tamaño de la muestra o número resultante de la adición del número de unidades que componen cada una de las dos muestras comparadas.

$n p$ : Número resultante del producto del número de unidades de cada una de las dos muestras comparadas.

$\overline{x_{a}}-\overline{x_{b}}$ : Diferencia entre las medias de ambas partes.

$S^{2}$ : Varianza de las dos muestras. ${ }^{17}$

$t$ : Desviación reducida «t de Student-Fisher». ${ }^{18}$

${ }^{17} \mathrm{Su}$ fórmula es: $S^{2}=\sigma_{a}^{2} n_{a}+\sigma_{b}^{2} n_{b} / N-2$. El concepto numérico $N-2\left(=n_{a}-1+n_{b}-1\right)$ corresponde en lenguaje estadístico al número de grados de libertad $(v)$, difícil concepto matemático que se ha de explicar mediante geometría vectorial. Los grados de libertad («degrees of freedom») en las pruebas para una muestra («one-sample test») equivalen a $n-1$, en nuestra caso al tratarse de una prueba «t de Student-Fisher» para dos muestras independientes («t test for two independent samples») los grados de libertad, por lo tanto, responden a $n_{a}-1+n_{b}-1$.

${ }^{18} \mathrm{Al}$ tratarse de muestras inferiores a 30 unidades $(\mathrm{n} \leq 30)$ se ha de aplicar este tipo de desviación reducida, cuya fórmula es la siguiente: $t=\left(\left|\overline{x_{a}}-\bar{x}_{b}\right| / \sqrt{ } S^{2}\right)(\sqrt{ } n p / N)$. 
La prueba estadística «t de Student-Fisher» también se puede representar mediante gráficos que nos brindan la posibilidad de apreciar de manera sinóptica la distribución de los distintos nexos. En pro de la claridad en los gráficos hemos agrupado los ocho

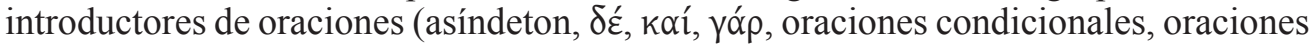
coordinadas, oraciones de relativo y oraciones subordinadas) en tres categorías: asíndeton, nexos coordinantes y nexos subordinantes. En el eje de abscisas (X) aparecen los grupos de 25 oraciones en los que se ha dividido cada sección y en el eje de ordenadas (Y) se registra el cómputo que arroja cada nexo en cada grupo.

El objetivo final de la prueba «t de Student-Fisher» es conocer el grado de probabilidad que tenemos para poder aceptar la hipótesis nula $\left(\mathrm{H}_{0}\right)$ o rechazarla, adoptando en tal caso la hipótesis alternativa $\left(\mathrm{H}_{1}\right)$. En nuestro caso la hipótesis nula consistiría en considerar los textos estudiados como obras de una misma población, que en el presente trabajo equivale a decir de un mismo autor, mientras que la hipótesis alternativa nos llevaría a adscribir los textos a diferentes autores. Esta probabilidad estadística se conoce como nivel de significación $\alpha$ y consiste en el riesgo que tenemos de incurrir en error al rechazar la hipótesis nula siendo ésta sin embargo verdadera. Por norma general, se suele admitir una posibilidad de error de $\alpha=0,05$ ó $5 \%$.

Como prueba suplementaria a la «t de Student-Fisher» se ha aplicado un análisis de rangos con el fin de calcular el coeficiente de correlación de los mismos. La correlación de Spearman o rho de Spearman $\left(r_{s}\right)$ es una medida de relación lineal entre dos variables cuyos datos son ordenados y reemplazados por su respectivo orden; es decir, se colocan los valores computados según el orden de aparición de cada uno de ellos y se le otorga un rango a cada valor. El coeficiente de correlación de Spearman, al igual que otros coeficientes de correlación como el de Pearson o el de Tau-b de Kendall, toma valores entre $-1 \mathrm{y}+1$ : un valor de +1 indica relación lineal perfecta positiva mientras que uno de -1 indica esta misma relación pero negativa, a su vez un valor de 0 indica relación lineal nula. Así pues el índice obtenido a través del análisis de rangos de Spearman será de +1 si hay identidad perfecta entre las dos clasificaciones lo que conlleva una unión muy fuerte; será de -1 si las dos clasificaciones son exactamente inversas; a saber, si el valor que tiene el primer rango en una clasificación es el último en la otra, mientras que el valor que ocupa el segundo lugar es el penúltimo en la otra serie y así sucesivamente; en tal caso aquí también existirá una unión muy fuerte e incluso una dependencia funcional. En último lugar, cuanto más próximo se encuentre el índice a 0 tanto más débil será la relación de dependencia.

En el análisis de Spearman la hipótesis nula $\left(\mathrm{H}_{0}\right)$ a diferencia de la prueba «t de Student-Fisher» conlleva la no interdependencia de las series, por esa razón cuanto más cerca de 0 esté el resultado final, más posibilidades tenemos de adoptar la hipótesis nula. Este análisis de rangos se distingue también de la prueba «t de Student-Fisher» en el hecho de que nos indica un dato de conjunto de las muestras, no un conjunto de tendencias en la distribución a lo largo de la muestra. ${ }^{19}$

Antes de finalizar estas premisas teóricas es preciso señalar un importante

${ }^{19}$ La aplicación del análisis de Spearman en detrimento de otros análisis como el de Pearson se debe a que hemos trabajado con un número de unidades en cada muestra inferior a 30; en estos casos se recomienda emplear Spearman. Cf. Woods, Fletcher y Hughes (1986: 173 s.) 
aspecto que se ha de calibrar al explicar los resultados obtenidos dado que existe una gran diferencia a la hora de valorar la no-desestimación de la hipótesis nula y su desestimación. El hecho de no desestimar la hipótesis nula no significa que ésta esté aceptada; sin embargo su rechazo exige la aceptación de la hipótesis alternativa.

Antes de adentrarnos en el estudio estadístico, es necesario hacer un breve paréntesis sobre la división en secciones del tratado: hemos adoptado la segmentación que proponía Galeno, seguida en parte por Fredrich (1899), según la cual los cc. 1-8 componían la primera sección, los cc. 9-15 la segunda y los cc. 16-22 la tercera,

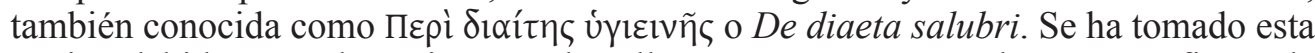
opción debido a que la crítica actual no llega a un consenso en lo que se refiere a la primera parte; así mientras que según Jouanna (1975) ésta consta de los cc. 1-7, para Schoene (1900) seguido por Deichgräber (1933) la primera sección incluiría hasta el capítulo noveno. Con nuestra decisión hemos intentado apartarnos lo más posible de juicios preestablecidos que pudieran mediatizar el análisis de la obra, además al aceptar una primera parte que acaba en el capítulo octavo adoptamos también una postura intermedia entre Jouanna y Schoene. Por otra parte para mayor claridad expositiva a lo largo del estudio estadístico nos referiremos a las diferentes secciones de la siguiente manera: $N H-1$ (cc. 1-8), $N H-2$ (cc. 9-15), $D S$ (cc. 16-22) y $N H$ en el caso de que estemos trabajando sobre los cc. 1-15 considerados como una unidad.

\section{LA PRESENTE INVESTIGACIÓN: APLICACIÓN PRÁCTICA}

La primera operación estadística a la que someteremos los datos será la prueba «t de Student-Fisher». Tal y como ya se ha señalado hemos procedido a dividir el texto de la siguiente manera: $\mathrm{NH}-1$ (cc. 1-8), $\mathrm{NH}-2$ (cc. 9-15), $D S$ (cc. 16-22).

El primer paso consistirá en conocer si las secciones $\mathrm{NH}-1$ y $\mathrm{NH}-2$ constituyen una unidad o si, por el contrario, pertenecen a autores distintos, como por ejemplo postulaba Galeno para quien los cc. 1-8 pertenecían a Hipócrates mientras que los cc. 9-15 eran la obra de un compilador de época helenística.

\section{Estadísticas de grupo}

\begin{tabular}{|lr|r|r|r|r|}
\hline & Grupos & $\mathrm{N}$ & Media & $\begin{array}{c}\text { Desviación } \\
\text { típica }\end{array}$ & $\begin{array}{c}\text { Error típico } \\
\text { de la media }\end{array}$ \\
\hline Asíndeton & 1 & 11 & 1,09 &, 944 &, 285 \\
& 2 & 7 &, 86 &, 690 &, 261 \\
\hline de & 1 & 11 & 6,36 & 3,264 &, 984 \\
& 2 & 7 & 6,29 & 2,498 &, 944 \\
\hline kai & 1 & 11 & 4,00 & 2,191 &, 661 \\
& 2 & 7 & 4,29 & 1,496 &, 565 \\
\hline gar & 1 & 11 & 2,55 & 1,368 &, 413 \\
& 2 & 7 & 2,14 & 1,215 &, 459 \\
\hline orcond & 1 & 11 & 1,64 & 1,690 &, 509 \\
& 2 & 7 &, 71 & 1,113 &, 421 \\
\hline orcond & 1 & 11 & 4,00 & 1,897 &, 572 \\
& 2 & 7 & 2,71 & 1,799 &, 680 \\
\hline orrel & 1 & 11 & 3,09 & 1,758 &, 530 \\
& 2 & 7 & 4,86 & 1,464 &, 553 \\
\hline orsub & 1 & 11 & 2,27 & 1,489 &, 449 \\
& 2 & 7 & 3,14 & 1,952 &, 738 \\
\hline
\end{tabular}




\section{Prueba de muestras independientes}

\begin{tabular}{|c|c|c|c|c|c|c|c|}
\hline & \multicolumn{2}{|c|}{$\begin{array}{c}\text { Prueba de Levene } \\
\text { para la igualdad } \\
\text { de varianzas }\end{array}$} & \multicolumn{4}{|c|}{ Prueba T para la igualdad de medias } \\
\hline & & $\mathrm{F}$ & Sig. & $\mathrm{t}$ & $\mathrm{gl}$ & $\begin{array}{c}\text { Sig. } \\
\text { (bilateral) }\end{array}$ & $\begin{array}{l}\text { Diferencia de } \\
\text { medias }\end{array}$ \\
\hline Asínd. & $\begin{array}{l}\text { Se han asumido } \\
\text { varianzas iguales } \\
\text {--------------- } \\
\text { No se han asumido } \\
\text { varianzas iguales }\end{array}$ & ,480 & ,498 & $\begin{array}{l}, 564 \\
, 606\end{array}$ & $\begin{array}{c}16 \\
15,559\end{array}$ & $\begin{array}{l}, 581 \\
, 554\end{array}$ & $\begin{array}{l}, 234 \\
, 234\end{array}$ \\
\hline de & $\begin{array}{l}\text { Se han asumido } \\
\text { varianzas iguales } \\
\text {--------------- } \\
\text { No se han asumido } \\
\text { varianzas iguales }\end{array}$ & ,479 & ,499 & $\begin{array}{l}, 054 \\
, 057\end{array}$ & $\begin{array}{c}16 \\
15,292\end{array}$ & $\begin{array}{l}, 958 \\
, 955\end{array}$ & $\begin{array}{l}, 078 \\
, 078\end{array}$ \\
\hline kai & $\begin{array}{l}\text { Se han asumido } \\
\text { varianzas iguales } \\
\text {----------------- } \\
\text { No se han asumido } \\
\text { varianzas iguales }\end{array}$ & 1,806 &, 198 & $\begin{array}{l}-, 302 \\
-, 329\end{array}$ & $\begin{array}{c}16 \\
15,845\end{array}$ & $\begin{array}{l}, 767 \\
, 747\end{array}$ & $\begin{array}{l}-, 286 \\
-, 286\end{array}$ \\
\hline gar & $\begin{array}{l}\text { Se han asumido } \\
\text { varianzas iguales } \\
-\begin{array}{l}\text { No se han asumido } \\
\text { varianzas iguales }\end{array}\end{array}$ &, 012 & ,914 & $\begin{array}{l}, 634 \\
, 652\end{array}$ & $\begin{array}{c}16 \\
14,089\end{array}$ & $\begin{array}{l}, 535 \\
, 525\end{array}$ & $\begin{array}{l}, 403 \\
, 403\end{array}$ \\
\hline $\begin{array}{l}\text { or. } \\
\text { cond. }\end{array}$ & $\begin{array}{l}\text { Se han asumido } \\
\text { varianzas iguales } \\
\text {----------------- } \\
\text { No se han asumido } \\
\text { varianzas iguales }\end{array}$ & 1,791 & ,199 & $\begin{array}{l}1,272 \\
1,396\end{array}$ & $\begin{array}{c}16 \\
15,937\end{array}$ & $\begin{array}{l}, 222 \\
, 182\end{array}$ & $\begin{array}{l}, 922 \\
, 922\end{array}$ \\
\hline $\begin{array}{l}\text { or. } \\
\text { coord. }\end{array}$ & $\begin{array}{l}\text { Se han asumido } \\
\text { varianzas iguales } \\
\text {----------------- } \\
\text { No se han asumido } \\
\text { varianzas iguales }\end{array}$ &, 016 & ,900 & $\begin{array}{l}1,429 \\
1,447\end{array}$ & $\begin{array}{c}16 \\
13,453\end{array}$ & $\begin{array}{l}, 172 \\
, 171\end{array}$ & $\begin{array}{l}1,286 \\
1,286\end{array}$ \\
\hline $\begin{array}{l}\text { or. } \\
\text { rel. }\end{array}$ & $\begin{array}{l}\text { Se han asumido } \\
\text { varianzas iguales } \\
\text {---------------- } \\
\text { No se han asumido } \\
\text { varianzas iguales }\end{array}$ & ,066 &, 801 & $\begin{array}{l}-2,209 \\
-2,305\end{array}$ & $\begin{array}{c}16 \\
14,659\end{array}$ & $\begin{array}{l}, 042 \\
, 036\end{array}$ & $\begin{array}{l}-1,766 \\
-1,766\end{array}$ \\
\hline $\begin{array}{l}\text { or. } \\
\text { sub. }\end{array}$ & $\begin{array}{l}\begin{array}{l}\text { Se han asumido } \\
\text { varianzas iguales }\end{array} \\
\text { - No se han asumido } \\
\text { varianzas iguales }\end{array}$ &, 038 & ,849 & $\begin{array}{l}-1,073 \\
-1,008\end{array}$ & $\begin{array}{c}16 \\
10,412\end{array}$ & $\begin{array}{l}, 299 \\
, 337\end{array}$ & $\begin{array}{l}-, 870 \\
-, 870\end{array}$ \\
\hline
\end{tabular}


Antes de analizar los datos, es necesario señalar que el SPSS realiza una prueba preliminar antes de realizar la prueba «t de Student-Fisher». ${ }^{20}$ Se trata de la prueba de Levene, cuyo resultado arroja un contraste (F) sobre la igualdad de varianzas que nos permite decidir si podemos suponer o no que las varianzas son homogéneas: si el contraste asociado al estadístico de Levene conlleva un nivel de significación mayor que 0,05 ó $5 \%$, entonces se habrá de suponer que las varianzas de las poblaciones son iguales por lo que en la prueba «t de Student-Fisher» habremos de atenernos a los datos que figuran en la primera línea, en la que -como reza el epígrafe- se han asumido varianzas iguales; si, por el contrario, el nivel de significación es menor que 0,05 habremos de asumir que las varianzas no son iguales por lo que tendremos que trabajar con los datos que aparecen en la segunda línea en el cuadro de la prueba «t de Student-Fisher». ${ }^{21}$

En el presente caso el estadístico de Levene corrobora que todas las varianzas son iguales, por lo que las ocho variables a tener en cuenta son las que aparecen registradas en la primera línea.

Así pues como muestran los datos aportados por la prueba «t de Student-Fisher» los niveles de significación se encuentran en todos los casos por encima del 0,05 ó $5 \%$, excepto en las oraciones de relativo donde tenemos un nivel de significación $\alpha=$ 0,042 . Este nivel por debajo del 0,05 se ha de poner en relación con la diferencia de medias que constatamos en el cuadro de estadísticos de grupo, donde podemos apreciar que dicha diferencia es la mayor que se registra en comparación con la media de las otras variables: 3,09 en $\mathrm{NH}-1$ ante 4,86 en $\mathrm{NH}-2$.

No obstante, teniendo en cuenta que incluso en el único caso donde nos encontramos por debajo del umbral del 5\% la probabilidad sólo alcanza el 4,2\% no parece oportuno desestimar la hipótesis nula por lo que, al menos en lo que respecta a la prueba «t de Student-Fisher», hemos de aceptar una única autoría.

A su vez, en los gráficos se han clasificado para mayor claridad los nexos en tres subgrupos: asíndeton, oraciones coordinadas ( $\delta \dot{\varepsilon}, k \alpha i ́$, үóp y nexos coordinantes), y oraciones subordinadas y de relativo. Un aspecto muy interesante, que los gráficos nos dan la oportunidad de advertir de manera visual, es la apreciación de que el empleo de la coordinación y de la subordinación son esencialmente antagónicos, de ahí que el aumento de uno de ellos conlleve el descenso del otro.

\footnotetext{
${ }^{20}$ Respecto a los cuadros es preciso advertir que por convención en el paquete informático SPSS no se anota la cifra 0 («cero») en los números que se encuentran entre $+1 /-1$; por consiguiente, un número como 0,12 aparecerá como, 12 .

${ }^{21}$ La igualdad de varianzas (homocedasticidad) se basa en la suposición de que la variable se distribuye con igual varianza en cualquiera de las estimaciones hechas mediante el modelo, por lo que constituye un criterio de homogeneidad. Por otra parte, cuando las varianzas no son iguales (heterocedasticidad), la prueba de Levene aplica determinadas operaciones matemático-estadísticas (fórmula de WelchSatterthwaite) que afectan a los grados de libertad pues éstos se han de adaptar a la asunción de varianzas no iguales, de ahí que en cada variable contemos con diferentes grados de libertad.
} 

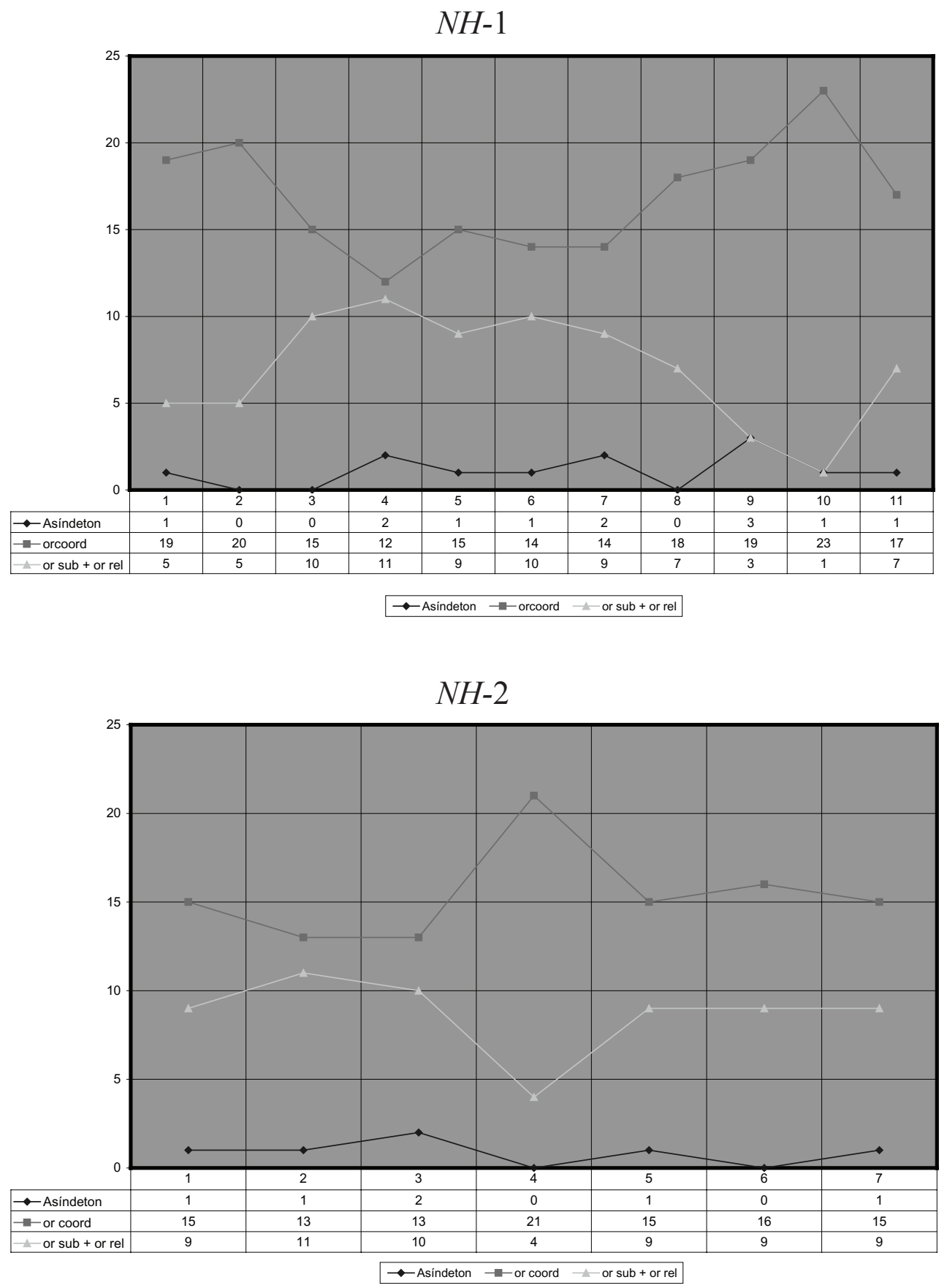

Estos gráficos nos permiten observar en ambas secciones una distribución paralela de los distintos nexos, correspondiendo a la coordinación la media más elevada, 
seguida de la subordinación y finalmente del asíndeton. No obstante, dentro de este paralelismo y del antagonismo evidente que presenta la coordinación respecto a la subordinación, el gráfico de $\mathrm{NH}-1$ demuestra que nos hallamos ante un estilo con una mayor variación tal y como queda reflejado en las oscilaciones que se reflejan en las líneas correspondientes a las medias de la coordinación y de la subordinación, mientras que la sección $\mathrm{NH}-2$ presenta una tendencia más estable, excepto en la zona central del gráfico donde sin embargo la desviación respecto a la tónica general no es tan marcada como en $\mathrm{NH}-1$, donde se constatan picos más acusados. Es decir, la mayor oscilación en $\mathrm{NH}-1$ refleja un uso más fluido en la sucesión de la coordinación y la subordinación, y una mayor alternancia de procedimientos, provocada quizá por el hecho de que $\mathrm{NH}-1$ constituya la sección más discursiva y doctrinal de las tres secciones estudiadas.

Asimismo, para refrendar los datos obtenidos con mayor seguridad se puede aplicar también el índice de rangos de Spearman:

\section{Correlaciones}

\begin{tabular}{|ccl|r|r|}
\hline & & NH1 & NH2 \\
\hline Rho Speaman & NH1 & Coeficiente de correlación & 1,000 &, $790^{*}$ \\
& & Sig. (bilateral) &, 020 \\
& N & 8 & 8 \\
\cline { 2 - 5 } & NH2 & Coeficiente de correlación &, $790^{*}$ & 1,000 \\
& Sig. (bilateral) &, 020 &. \\
& N & 8 & 8 \\
\hline
\end{tabular}

* La correlación es significativa al nivel 0,05 (bilateral)

Así pues, el coeficiente $\mathrm{r}_{\mathrm{s}} \mathrm{o}$ rho de Spearman ${ }^{22}$ se encuentra por debajo del 0,05 ó 5\%, más exactamente en el 0,020 según los valores significativos que ofrece el paquete informático SPSS. Por lo tanto, el análisis de rangos de Spearman nos permite desestimar la hipótesis nula de no interdependencia, por lo que nos encontramos ante textos relacionados entre sí.

En suma, tanto la prueba «t de Student-Fisher» como el análisis de correlación de rangos de Spearman han demostrado claramente que las secciones $\mathrm{NH}-1$ (cc. 1-8) y $\mathrm{NH}$-2 (cc. 9-15) pertenecen a un mismo autor.

Pasemos por lo tanto a comparar la tercera sección o $D S$ (cc. 16-22) conocida como

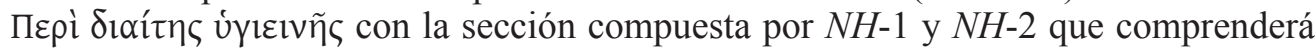
los cc. 1-15, una vez corroborada su unidad.

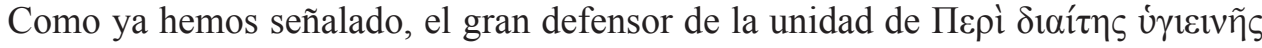
con el resto del tratado es Jouanna (1975) en contra de la opinión expresada en su momento por Littré y seguida entre otros por Jones, según la cual dicho opúsculo era independiente.

Antes de pasar a realizar los cómputos estadísticos hay que señalar que en $\mathrm{NH}$-tomado en su totalidad- contamos con 19 muestras, a pesar de que las secciones $\mathrm{NH}$-1

${ }^{22}$ El coeficiente $r$ ho de Spearman obedece a la siguiente fórmula: $r_{s}=1-6 \Sigma \mathrm{d}^{2} / \mathrm{n}\left(\mathrm{n}^{2}-1\right)$. 
y $\mathrm{NH}-2$ arrojaban 11 y 7 muestras respectivamente. El hecho de contabilizar una serie más de 25 oraciones se debe a que en la serie 19 se han incluido las oraciones de las secciones $N H-1$ y $N H-2$ que habían resultado sobrantes en el primer recuento debido a que su número no era suficiente para formar una serie de 25 .

Estadísticas de grupo

\begin{tabular}{|lr|r|r|r|r|}
\hline & Grupos & $\mathrm{N}$ & Media & $\begin{array}{c}\text { Desviación } \\
\text { típica }\end{array}$ & $\begin{array}{c}\text { Error típico } \\
\text { de la media }\end{array}$ \\
\hline Asíndeton & 1 & 19 & 1,11 &, 937 &, 215 \\
& 2 & 6 & 1,83 & 1,722 &, 703 \\
\hline de & 1 & 19 & 6,53 & 2,951 &, 677 \\
& 2 & 6 & 6,67 & 1,506 &, 615 \\
\hline kai & 1 & 19 & 4,00 & 1,915 &, 439 \\
& 2 & 6 & 7,00 & 1,265 &, 516 \\
\hline gar & 1 & 19 & 2,32 & 1,293 &, 297 \\
& 2 & 6 & 3,17 & 2,229 &, 910 \\
\hline orcond & 1 & 19 & 1,21 & 1,512 &, 347 \\
& 2 & 6 &, 17 &, 408 &, 167 \\
\hline orcond & 1 & 19 & 3,37 & 1,950 &, 447 \\
& 2 & 6 & 1,50 & 1,378 &, 563 \\
\hline orrel & 1 & 19 & 3,84 & 1,803 &, 414 \\
& 2 & 6 & 2,50 & 1,643 &, 671 \\
\hline orsub & 1 & 19 & 2,63 & 1,640 &, 376 \\
& 2 & 6 & 2,17 & 2,137 &, 872 \\
\hline
\end{tabular}




\section{Prueba de muestras independientes}

\begin{tabular}{|c|c|c|c|c|c|c|c|}
\hline & \multicolumn{2}{|c|}{$\begin{array}{l}\text { Prueba de Levene } \\
\text { para la igualdad de } \\
\text { varianzas }\end{array}$} & \multicolumn{4}{|c|}{ Prueba T para la igualdad de medias } \\
\hline & & $\mathrm{F}$ & Sig. & $\mathrm{t}$ & $\mathrm{gl}$ & $\begin{array}{c}\text { Sig. } \\
\text { (bilateral) }\end{array}$ & $\begin{array}{l}\text { Diferencia } \\
\text { de medias }\end{array}$ \\
\hline Asínd. & \begin{tabular}{|l}
$\begin{array}{l}\text { Se han asumido } \\
\text { varianzas iguales }\end{array}$ \\
$-\begin{array}{l}\text { No se han asumido } \\
\text { varianzas iguales }\end{array}$ \\
\end{tabular} & 8,467 &, 008 & $\begin{array}{l}-1,347 \\
-, 990\end{array}$ & $\begin{array}{c}23 \\
5,963\end{array}$ & $\begin{array}{l}, 191 \\
, 361\end{array}$ & $\begin{array}{l}-, 728 \\
-, 728\end{array}$ \\
\hline de & $\begin{array}{l}\begin{array}{l}\text { Se han asumido } \\
\text { varianzas iguales }\end{array} \\
\text { No se han asumido } \\
\text { varianzas iguales }\end{array}$ & 5,815 &, 024 & $\begin{array}{l}-, 111 \\
-, 153\end{array}$ & $\begin{array}{c}23 \\
17,383\end{array}$ & $\begin{array}{l}, 913 \\
, 880\end{array}$ & $\begin{array}{l}-, 140 \\
-, 140\end{array}$ \\
\hline kai & $\begin{array}{l}\begin{array}{l}\text { Se han asumido } \\
\text { varianzas iguales } \\
\text { - } \\
\text { No se han asumido } \\
\text { varianzas iguales }\end{array} \\
\end{array}$ & 1,704 & ,205 & $\begin{array}{l}-3,572 \\
-4,425\end{array}$ & $\begin{array}{c}23 \\
12,969\end{array}$ & $\begin{array}{l}, 002 \\
, 001\end{array}$ & $\begin{array}{l}-3,000 \\
-3,000\end{array}$ \\
\hline gar & $\begin{array}{l}\begin{array}{l}\text { Se han asumido } \\
\text { varianzas iguales }\end{array} \\
------------- \\
\text { No se han asumido } \\
\text { varianzas iguales }\end{array}$ & 4,632 &, 042 & $\begin{array}{l}-1,176 \\
-, 889\end{array}$ & $\begin{array}{c}23 \\
6,101\end{array}$ & $\begin{array}{l}, 252 \\
, 408\end{array}$ & $\begin{array}{l}-, 851 \\
-, 851\end{array}$ \\
\hline $\begin{array}{l}\text { or. } \\
\text { cond. }\end{array}$ & $\begin{array}{l}\begin{array}{l}\text { Se han asumido } \\
\text { varianzas iguales }\end{array} \\
--------- \\
\text { No se han asumido } \\
\text { varianzas iguales }\end{array}$ & 4,922 &, 037 & $\begin{array}{l}1,650 \\
2,712\end{array}$ & $\begin{array}{c}23 \\
22,880\end{array}$ & $\begin{array}{l}, 113 \\
, 012\end{array}$ & $\begin{array}{l}1,044 \\
1,044\end{array}$ \\
\hline $\begin{array}{l}\text { or. } \\
\text { coord. }\end{array}$ & $\begin{array}{l}\begin{array}{l}\text { Se han asumido } \\
\text { varianzas iguales }\end{array} \\
-\begin{array}{l}\text { No se han asumido } \\
\text { varianzas iguales }\end{array} \\
\end{array}$ & 1,479 & ,236 & $\begin{array}{l}2,168 \\
2,599\end{array}$ & $\begin{array}{c}23 \\
11,985\end{array}$ & $\begin{array}{l}, 041 \\
, 023\end{array}$ & $\begin{array}{l}1,868 \\
1,868\end{array}$ \\
\hline $\begin{array}{l}\text { or. } \\
\text { rel. }\end{array}$ & $\begin{array}{l}\text { Se han asumido } \\
\text { varianzas iguales } \\
\text {------------- } \\
\text { No se han } \\
\text { asumido } \\
\text { varianzas iguales }\end{array}$ & .002 & ,962 & $\begin{array}{l}1,620 \\
1,703\end{array}$ & $\begin{array}{c}23 \\
9,158\end{array}$ & $\begin{array}{l}, 119 \\
, 122\end{array}$ & $\begin{array}{l}1,342 \\
1,342\end{array}$ \\
\hline $\begin{array}{l}\text { or. } \\
\text { sub. }\end{array}$ & $\begin{array}{l}\text { Se han asumido } \\
\text { varianzas iguales } \\
\text { - No se han asumido } \\
\text { varianzas iguales }\end{array}$ &, 284 & ,599 & $\begin{array}{l}, 564 \\
, 489\end{array}$ & $\begin{array}{c}23 \\
6,966\end{array}$ & $\begin{array}{l}, 578 \\
, 640\end{array}$ & $\begin{array}{l}, 465 \\
, 465\end{array}$ \\
\hline
\end{tabular}


Lo primero que advertimos al observar la tabla de las pruebas de Levene y «t de Student-Fisher» es que en cuatro de las ocho variables (asíndeton, $\delta \varepsilon$, үớ $\rho$ y or. condicionales), la prueba de Levene presenta un contraste $(F)$ cuyo nivel de significación está por debajo del $5 \%$, lo cual indica que se ha de rechazar la hipótesis de igualdad de varianzas por lo que en estos casos, cuando las varianzas no son iguales, el SPSS calcula los grados de libertad mediante la aproximación de Welch-Satterthwaite. Como se puede apreciar, el nuevo valor de los grados de libertad es menor que el anterior porque se pierde precisión debido a la desigualdad de las varianzas (o heterocedasticidad).

No obstante, dicha desigualdad de varianzas parece no tener ninguna relación con la aceptación o rechazo de la hipótesis nula en los resultados de la prueba «t de StudentFisher». Así de los cuatro casos de heterocedasticidad tan sólo se ha de rechazar la hipótesis nula en la variable de las oraciones condicionales donde atestiguamos un nivel de significación $\alpha=0,012 ;{ }^{23}$ por lo que, al menos en esta variable, habríamos de postular la hipótesis alternativa $\left(\mathrm{H}_{1}\right)$. Sin embargo, en las tres variables restantes

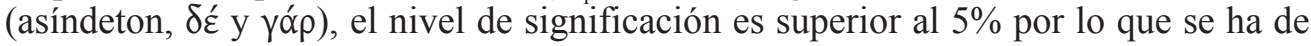
aceptar la hipótesis nula $\left(\mathrm{H}_{0}\right)$.

Tras haber tratado los casos de desigualdad de varianzas, pasemos a continuación a las cuatro variables cuyos resultados se encuentran en el marco de la igualdad de varianzas (homocedasticidad).

Según la tabla se ha de rechazar la hipótesis nula en la variable кaí y en las oraciones coordinadas pues arrojan un nivel de significación del 0,002 y del 0,041. Este nivel de significación por debajo del $5 \%$ está en consonancia con la diferencia entre las medias correspondiente que son las mayores que se registran en las tablas comparativas de los estadísticos de grupo de $N H$ y $D S$. Así kaí presenta una media de 4,00 en $\mathrm{NH}$ ante una de 7,00 en $\mathrm{DS}$, mientras que las oraciones coordinadas arrojan una media de 3,37 en $N H$ y de 1,50 en $D S$. Esta diferencia de 3 puntos entre las medias de кaí en los dos textos comparados provoca que el nivel de significación de la prueba «t de Student-Fischer» alcance el 0,2\%, el menor de todos los niveles de significación registrados.

Por otra parte la media de $\alpha \alpha i ́(7,00)$ en $D S$ es la mayor que se aprecia tanto en $D S$, como en $N H$ (tomado en su totalidad o dividido en sus dos partes); este hecho nos está indicando que en $D S$ prima un estilo paratáctatico basado en la conjunción koí en detrimento de otros nexos. Probablemente por esta causa, la prueba «t de StudentFisher» arroja también un nivel de significación inferior al 0,05 en el apartado de la coordinación donde se han incluido otros procedimientos paratácticos al margen de

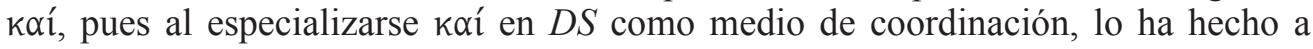
expensas de los restantes nexos coordinantes. Este hecho queda refrendado al comprobarse que en la variable de las oraciones coordinadas la media mayor se encuentra en $N H$ (3,37 ante 1,50 en $D S)$, lo cual corrobora que mientras que en $D S$ la coordinación se ha fundamentado básicamente en koí, en $N H$ nos hallamos ante una parataxis con mayor riqueza de medios expresivos. Esta particularidad se advierte claramente en

${ }^{23}$ Recordemos que cuando la prueba de Levene no asume la igualdad de varianzas, los datos que se han de consultar son los de la fila inferior, los correspondientes a la heterocedasticidad. 


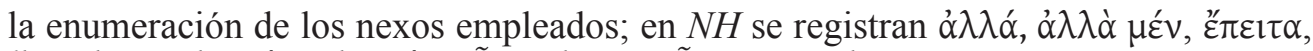

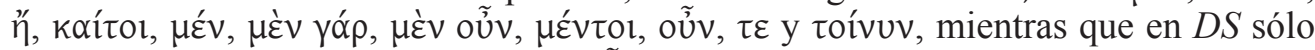

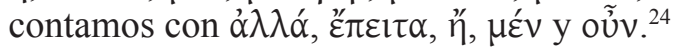

En suma, de las ocho variables analizadas la prueba «t de Student-Fisher» indica que se ha de rechazar la hipótesis nula en tres (кaí, or. condicionales y or. coordinadas); por lo tanto este hecho nos permitiría plantearnos en un primer momento la posibilidad de rechazar en su totalidad la hipótesis nula a la espera de los datos que suministran las restantes pruebas. ${ }^{25}$

Pasemos así pues, a comprobar si los gráficos arrojan alguna luz en pro o en contra de la unidad de $N H$ respecto a $D S$, partiendo siempre de la base de que los gráficos sólo nos pueden ofrecer una visión aproximada de la distribución de los diferentes nexos.

\section{$N H$}

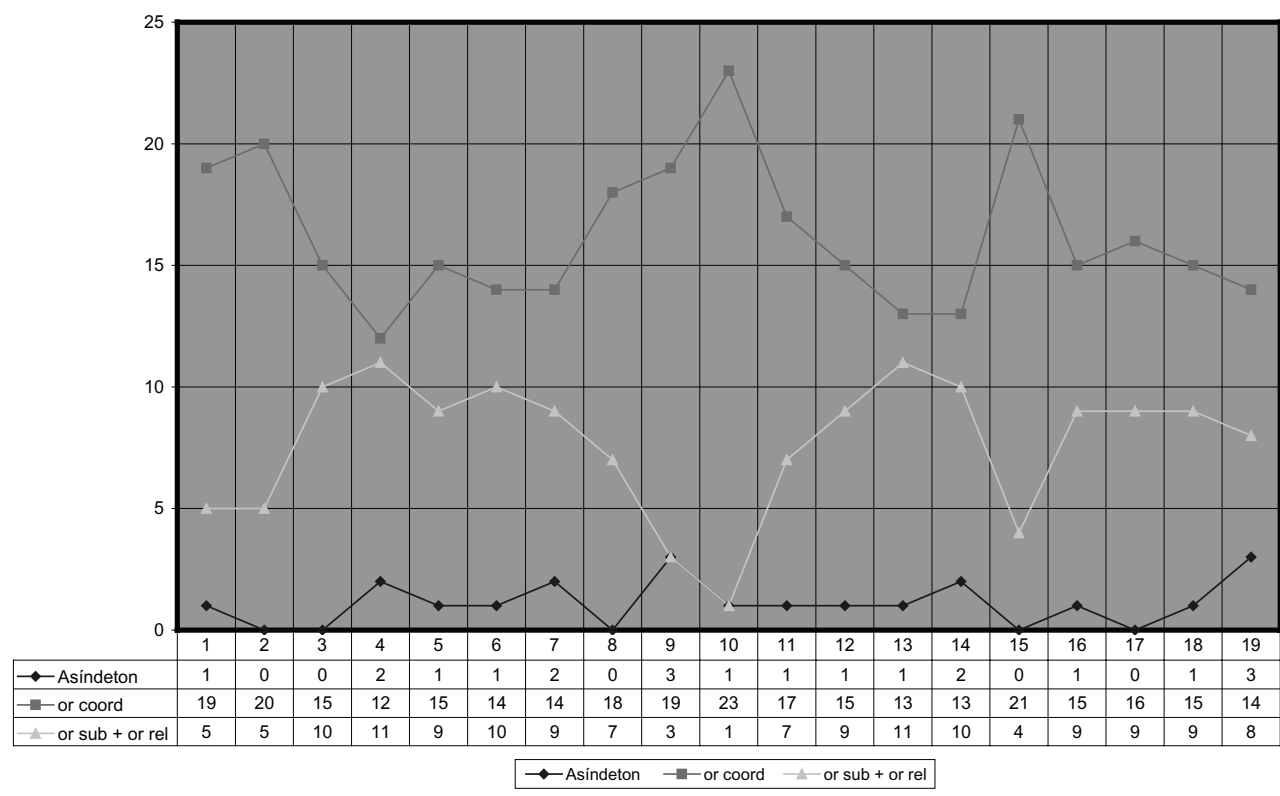

${ }^{24}$ Sierra de Grado (2003: 413) incluye кaítot entre las oraciones coordinadas, igualmente Rodríguez

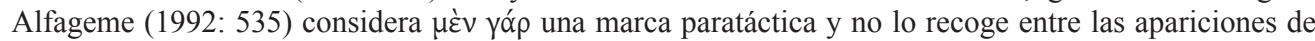

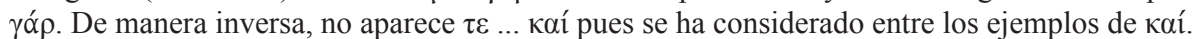

${ }^{25}$ Nuestra hipótesis de trabajo está en consonancia con el método de Labiano Ilundáin (2004) quien, basándose en tres variables (or. coordinadas, or. subordinadas y de relativo, y asíndeton), rechaza sistemáticamente la hipótesis nula en todos los casos en que una de las tres variables está por debajo del 0,05. 


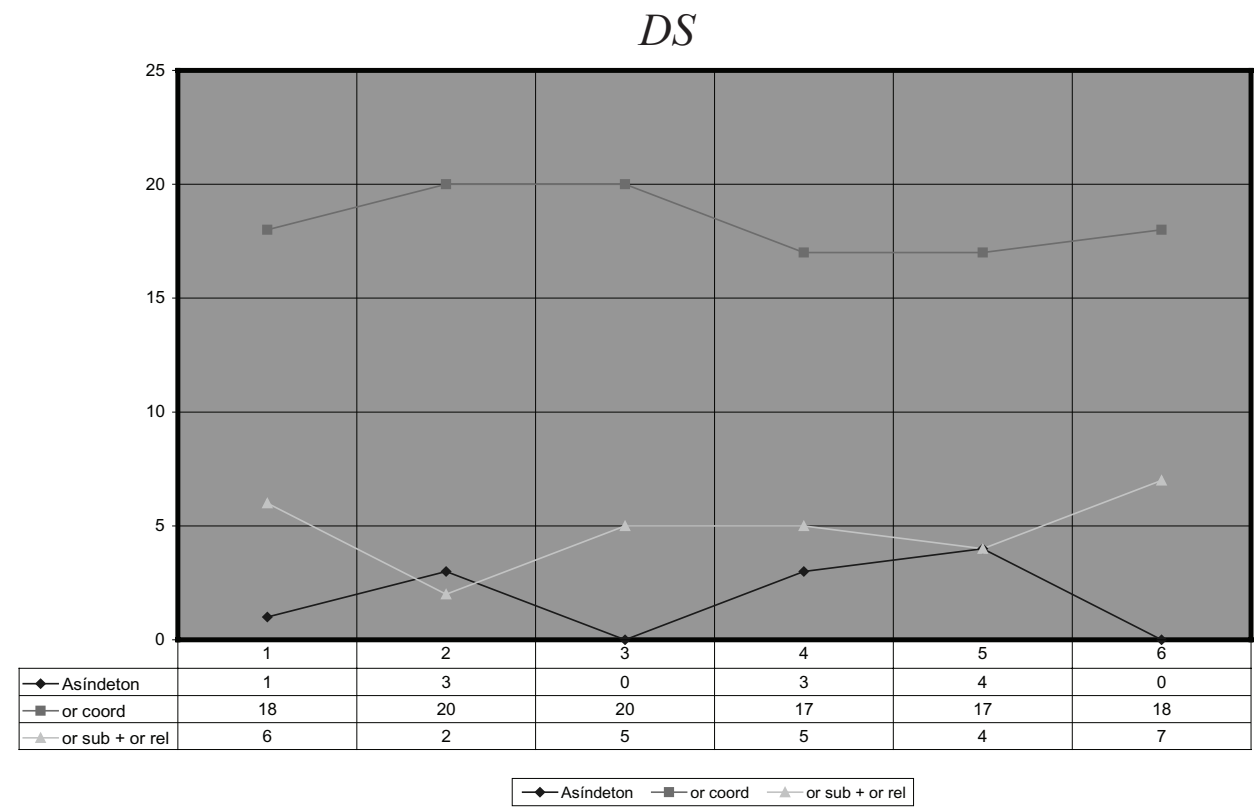

A pesar de que los gráficos no constituyen una prueba firme, es evidente que una comparación sinóptica de ambos parece demostrar que nos hallamos ante estilos bastante

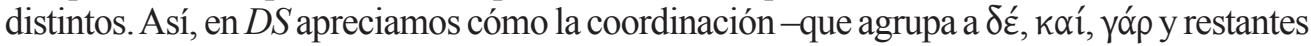
nexos coordinantes- se destaca del resto de las variables y presenta un diseño bastante homogéneo, homogeneidad que se observa en el conjunto de $D S$. Por el contrario, el gráfico de $\mathrm{NH}$ tiene una disposición mucho más accidentada, lo cual probablemente esté en consonancia con el hecho de que $\mathrm{NH}$ haga gala de un estilo más variado en el que alternan los períodos subordinados con la coordinación, mientras que en $D S$ prima la parataxis.

No obstante, las divergencias entre ambos gráficos no suponen una prueba palmaria de la diferente autoría de ambos textos, sólo de la variedad de estilos, que puede estar provocada por múltiples factores, como por ejemplo la temática, dado que $\mathrm{NH}$ es mucho más discursivo que $D S$, donde nos encontramos sobre todo ante una serie de prescripciones médicas. Por lo tanto, se hace necesario apelar a nuevas pruebas estadísticas, como la de Spearman, para saber si tales diferencias nos conducen al rechazo o a la aceptación de una misma autoría.

\section{Correlaciones}

\begin{tabular}{|c|c|c|c|c|}
\hline & & & NH1 & $\mathrm{NH} 2$ \\
\hline \multirow[t]{2}{*}{ Rho Speaman } & NH1 & $\begin{array}{l}\text { Coeficiente de correlación } \\
\text { Sig. (bilateral) } \\
\mathrm{N}\end{array}$ & $\begin{array}{r}1,000 \\
8 \\
8\end{array}$ & $\begin{array}{r}690 \\
, 058 \\
8 \\
\end{array}$ \\
\hline & $\mathrm{NH} 2$ & $\begin{array}{l}\text { Coeficiente de correlación } \\
\text { Sig. (bilateral) } \\
\text { N }\end{array}$ & $\begin{array}{r}, 690 \\
, 058 \\
8\end{array}$ & $\begin{array}{r}1,000 \\
8\end{array}$ \\
\hline
\end{tabular}


El coeficiente de Spearman arroja un nivel de significación ligeramente por encima del 0,05, exactamente del 0,058. Por consiguiente, el análisis de rangos de Spearman no nos permite descartar la hipótesis nula de no interdependencia de las series; por lo tanto, nos encontraríamos ante dos textos no relacionados entre sí, al menos en lo que respecta al análisis de Spearman.

En suma, en la prueba «t de Student-Fisher» tres de las ocho variables presentan un nivel de significación por debajo del 5\% lo que conlleva, al menos en estos casos, el rechazo de la hipótesis nula de dependencia o interrelación; a su vez, el análisis de Spearman nos induce a pensar que hemos de aceptar la hipótesis nula de independencia. ${ }^{26}$ Por lo tanto, la unión de estas dos pruebas nos capacita para plantearnos la posibilidad de que $N H$ y $D S$ sean textos independientes. No obstante, hemos de adoptar una cierta precaución dado que en la prueba «t de Student-Fisher» sólo tres variables desestiman hipótesis nula y que el nivel de significación de Spearman es sólo levemente superior al $5 \%$, más exactamente el $5 \%$.

De todas maneras, la suma de los resultados de ambas pruebas si bien no constituye una confirmación categórica de la independencia de ambos tratados, sí permite formular muy serias dudas respecto a la visión monolítica de su unidad, a pesar de haber sido postulada por eruditos de la talla de Jouanna y de contar en la actualidad con el beneplácito de la comunidad filológica.

\section{BIBLIOGRAFÍA}

Alsina i Clota, J. (1974), «Deux notes hippocratiques», Studii Clasice, 16, 211-216.

- (1983), Hipòcrates. Tractats mèdics. Volum III: Sobre la naturalesa de l'home, Epidèmies I $i$ III, Barcelona.

Ángel y Espinós, J. (2003), Comentario sintáctico-estilístico de Epidemias V y VII, (Tesis doctoral), Madrid.

URL: http://www.ucm.es/BUCM/tesis/19972000/H/3/H3052101.pdf

Deichgräber, K. (1933), Die Epidemien und das Corpus Hippocraticum. Voruntersuchungen zu einer Geschichte der koischen Ärtzeschule, Berlin 1933, reimpr. Berlin-New York 1971.

FREDRICH, C. J. (1899), Hippokratische Untersuchungen. Die Lehre von den vier Temperamenten, Berlin 1899; reimpr. New York 1976.

Grensemann, H. (1974), «Polybos (8)», en Paulys Realencyclopädie der classischen Altertumswissenschaft, Suppl. 14, cols. 428-436.

Jones, W. H. S. (1931), Hippocrates, vol. IV, Cambridge (Massachusetts)-London, 1931; reimpr. 1959.

- (1947), The Medical Writings of Anonymus Londinensis, translated and edited, Cambridge 1947, reimpr. 1968.

${ }^{26}$ Recordemos que en el análisis de rangos de Spearman la hipótesis nula $\left(\mathrm{H}_{0}\right)$ conlleva la no interdependencia de las series, al contrario de la prueba «t de Student-Fisher» donde la hipótesis nula supone la interrelación de las series. 
Jounnna, J. (1969), «Le médecin Polybe, est-il l'auteur de plusieurs ouvrages de la Collection hippocratique?», REG 82, 552-562.

- (1975), Hippocrate, La nature de l’homme, édité, traduit et commenté, Berlin.

KüHn, C. G. (1821-1833), Galeni opera omnia, 20 vols., Leipzig.

Labiano Ilundáin, J. M. (2004), «Los tratados quirúrgicos del Corpus Hippocraticum. Aplicación de análisis de estadística lingüística», $C F C$ (egi) 14, 91-109.

LitTré, É. (1839-1861), Oeuvres complètes d'Hippocrate, 10 vols., Paris 1839-1861, reimpr. Amsterdam 1961-1962.

Mewaldt, J. (1914), Galeni In Hippocratis De natura hominis commentaria tria, LeipzigBerlin, 1-113, en A.A. V.V., Galeni in Hippocratis De natura hominis, in Hippocratis De victu acutorum, De diaeta Hippocratis in morbis acutis, Leipzig-Berlin.

Müller, CH. (1973), Estadísitica lingüística, Madrid 1973. Traducción española de A. Quilis de Initiation à la statistique linguistique, Paris 1968.

Rietveld, T. y van Hout, R. (2005), Statistics in Language Research: Analysis of Variance, Berlin-New York.

Rodríguez Alfageme, I. (1992), «Sobre la fecha de Hipp. De glandulis», Epos 8, 549-566.

— (1993): «La atribución de Hipp. De visu», CFC (egi) 3, 57-65.

Schoene, H. (1900): Reseña a Fredrich (1899), Göttingische gelehrte Anzeigen, 157, 654-662.

Sierra De Grado, C. (2003): Rasgos de estilo y recursos de composición en algunos tratados del Corpus Hippocraticum, (Tesis doctoral), Madrid.

URL: http://www.ucm.es/BUCM/tesis/19972000/H/3/H3073601.pdf

Villaret, O. (1911), Hippocratis de Natura hominis liber ad codicum fidem recensitus, dissertatio inauguralis (...). Accedunt prolegomena de codicibus et de dialecto hujus libri et adnotationes, Berlin - Göttingen.

Woods, A., Fletcher, P. y Hughes, A. (1986): Statistics in Language Studies, Cambridge 1986, reimpr. 1989. 